(2) Open Access Full Text Article

REVIEW

\title{
Systemic Approach to Recurrent Primary CNS Lymphoma: Perspective on Current and Emerging Treatment Strategies
}

This article was published in the following Dove Press journal: OncoTargets and Therapy

\author{
Matthias Holdhoff ${ }^{\prime}$ \\ Nina Wagner-Johnston' \\ Mark Roschewski $\mathbb{I D}^{2}$ \\ 'Department of Oncology, The Sidney \\ Kimmel Comprehensive Cancer Center \\ at Johns Hopkins, Baltimore, Maryland, \\ USA; ${ }^{2}$ Lymphoid Malignancies Branch, \\ National Cancer Institute, Bethesda, \\ Maryland, USA
}

\begin{abstract}
There is no uniform standard of care for the treatment of refractory or recurrent primary central nervous lymphoma ( $\mathrm{r} / \mathrm{r}$ PCNSL). Many different systemic treatment regimens have been studied, but available data are based on small prospective or retrospective reports. There have been no randomized controlled trials in $r / r$ PCNSL to date. Here, we provide an overview of published systemic regimens for the treatment of $r / r$ PCNSL, as well as therapies that are under investigation. In addition, based on available data, we propose strategies of how to approach choice of therapy for different groups of patients in this disease setting. Patients can be mainly divided into three groups: 1) patients suitable for a rechallenge with high-dose methotrexate (HD-MTX)-based regimens and that may or may not be candidates for consolidation with high-dose chemotherapy with autologous stem cell transplant, 2) patients refractory to HD-MTX or that had early relapse, but suitable for an aggressive treatment strategy with re-induction with non-MTX-based therapy, possibly followed by high-dose chemotherapy with autologous transplant, and 3) patients not suitable for re-treatment with HD-MTX and that are not candidates for aggressive therapy. As PCNSL is a rare disease and as there is urgent need for better outcomes in $r / r$ PCNSL, clinical trial participation is encouraged, especially in elderly or frail patients who are not candidates for high-dose chemotherapy and transplant.
\end{abstract}

Keywords: primary central nervous system lymphoma, PCNSL, recurrent PCNSL, B-cell lymphoma, ibrutinib, autologous stem cell transplant

\section{Introduction}

Diffuse large B-cell lymphomas (DLBCL) are aggressive B-cell lymphomas that may involve the central nervous system (CNS). Primary DLBCL of the CNS or primary central nervous system lymphoma (PCNSL) refers to a rare subtype of DLBCL involving the brain, eyes, leptomeninges, or spinal cord without evidence of extracranial systemic involvement. Primary intraocular lymphoma (PIOL) is a subtype of PCNSL that presents in the retina, vitreous chamber and/or the optic nerve and may have concurrent involvement at other sites within the CNS. PCNSL has an estimated annual incidence of 1500 cases in the United States accounting for $6 \%$ of all newly diagnosed malignant brain tumors. ${ }^{1,2}$ The incidence of PCNSL is highest among males and is currently rising in immunocompetent patients, particularly those with advanced age. ${ }^{3-5}$ Secondary DLBCL of the CNS or secondary CNS lymphoma (SCNSL) can be distinguished from PCNSL by either isolated relapse of DLBCL within the CNS or synchronous
Correspondence: Matthias Holdhoff Email mholdhol@jhmi.edu 
CNS and systemic involvement. Relapse of DLBCL within the CNS is a feared complication that may arise shortly after, or even during frontline therapy. ${ }^{6,7}$ As a result, prophylactic strategies focus on prevention of SCNSL during frontline therapy in high-risk subgroups of DLBCL. Patients with SCNSL and isolated CNS relapse are often treated as PCNSL, while patients with synchronous CNS and systemic disease pose a clinical dilemma. $^{8}$

The molecular heterogeneity of DLBCL is divided by gene-expression profiling (GEP) into two major molecular subtypes based on their cell of origin: germinal center B-cell (GCB) and activated B-cell (ABC) subtypes. ${ }^{9-11}$ Studies in PCNSL have demonstrated that the majority of cases have gene expression profiles of $\mathrm{ABC}$ DLBCL. ${ }^{12}$ Further, genomic characterization studies in PCNSL have demonstrated mutations in MYD88 ${ }^{\mathrm{L} 265 \mathrm{P}}$ and CD79B, often seen in cases of ABC DLBCL. ${ }^{13-15}$ Historically, PCNSL has been considered a distinct entity from systemic DLBCL, but biologic links are evident. Comprehensive molecular characterization of DLBCL demonstrates genetic subtypes that frequently involve extranodal sites including the CNS and testis. ${ }^{16,17}$ Further, a number of targetable genetic features have been identified in DLBCL subsets that frequently invade the CNS. ${ }^{16}$ Improved understanding of the shared molecular biology between PCNSL and SCNSL along with novel agents that reliably penetrate CNS tumors has introduced a host of new therapeutic targets. ${ }^{18-20}$ In this review, we aim to discuss the current treatment landscape of these CNS lymphomas with an emphasis on treatment strategies for recurrent PCNSL.

High-dose methotrexate (HD-MTX) is the backbone for the treatment of newly-diagnosed PCNSL and also secondary CNS lymphomas. There are several treatment regimens with HD-MTX that have shown significant efficacy in newly diagnosed PCNSL that significantly vary regarding HD-MTX dose, paired drugs and treatment schedules. $^{2}$ Most of the different regimens have not been prospectively compared and it is not possible to conclusively state which of the regimens is the most effective. In addition, there has remained controversy about which type of consolidation regimen may be most beneficial after induction with MTX-based induction therapy. Trials that evaluated more aggressive upfront regimens typically select for patients with comparatively good performance status and these therapies may not be appropriate for many newly diagnosed patients, including the growing number of elderly patients with PCNSL. The focus of this article is not on efficacy and comparison of upfront therapies, but on treatment options for patients with progressive or recurrent disease.

Despite significant efficacy of regimens for newly diagnosed PCNSL, most patients eventually relapse and require additional therapy. Relapses can be seen late, even over 10 years after initial therapy. ${ }^{21,22}$ There is a difference though between patients whose tumors do not respond to HD-MTX-based therapy (primary refractory patients) or patients who recur early (eg, within half a year from achieving a CR) and those whose tumors recur after having achieved a durable CR with MTX-based therapy. For the former, changing therapy to a non-MTX-based therapy is required, whereas for patients that had initially benefited from MTX-based therapy, a re-challenge with the same or a similar MTX-based treatment may be most reasonable. Over the past 15 years, there has been a growing body of evidence of treatment options for progressive or recurrent PCNSL. $^{23,24}$ This includes data about repeat treatment with MTX-based therapy as well as new drugs. In addition, fit patients who achieve a CR or good response to reinduction therapy may be candidates for high-dose therapy with autologous stem cell transplant. For patients not suitable for autologous transplant, consolidation with lowdose radiation is another option for consolidation that can be considered. ${ }^{25}$

\section{Repeat Therapy with HD-MTX-Based Therapy}

Repeat treatment with HD-MTX can be highly effective in patients with that had previously achieved a CR with HDMTX-based regimens. Data of 22 patients, 19 of which had been treated with HD-MTX monotherapy at initial diagnosis and that had achieved a CR was re-challenged with HDMTX monotherapy ( $\geq 3 \mathrm{~g} / \mathrm{m}^{2}$ ). The overall response rate in this study was $91 \%$ to first salvage therapy with CR in 16 (73\%) patients, 2 patients with a PR and one with a mixed response. Data showed that even a second salvage therapy may be effective in these patients. ${ }^{26}$ Similarly, another retrospective study from Memorial Sloan Kettering Cancer Center on 39 patients with relapsed PCNSL after initial response to HD-MTX-based therapy with median time from initial diagnosis of 26 months (range, 8.7-178 months) with an objective response rate of $85 \%$ and $\mathrm{CR}$ rate of $74 \%$ and a $10 \%$ PR. Thirty-seven of the patients had received methotrexate, procarbazine and vincristine (MPV) 
as their initial therapy. Different regimens were used at MTX re-challenge, the majority of which were MPV and rituximab (44\%) and MPV (23\%), but also single-agent MTX (15\%) and MTX, BCNU and etoposide (10\%). Median progression free survival was 16 months, median overall survival 41 months and 1-year overall survival $79 \% .{ }^{27}$ As different combination therapies that are used for initial induction appear to be effective for this patient population, this also provides a rationale for the development for additional combinations of therapy with MTX in the recurrent setting. A recently published combination regimen of MTX and ibrutinib showed promising activity. ${ }^{28}$

There currently are no data that determine a minimum duration of $\mathrm{CR}$ to determine when patients would be candidates for repeat treatment with HD-MTX versus another treatment approach. The National Comprehensive Cancer Network (NCCN) guidelines are currently using a cut off of 12 months as a divider ( $<12$ months defined as short duration, $\geq 12$ months defined as long duration of previous response) (NCCN.org). Given the lack of clear evidence regarding a specific minimum time interval from initial CR, clinical judgement is still required to guide selection of patients who may be best suited for a rechallenge with HD-MTX-based therapy.

\section{Non-HD-MTX-Based Therapies}

For patients that are not suitable for a re-challenge with HD-MTX, there are data on a number of single agent and combination regimens that encompass a broad variety of mechanisms of action. The different regimens are summarized in Table 1.

\section{Temozolomide}

Temozolomide (TMZ) has been studied in recurrent PCNSL alone or in combination with rituximab. ${ }^{29-33}$ In a prospective Phase II study with 36 patients with recurrent PCNSL after HD-MTX-containing therapy and/or radiotherapy, monotherapy with TMZ showed an objective response rate (ORR) of $31 \%$, with 9 complete and 2 partial responses. ${ }^{29}$ A second study with 17 patients within part heavily pretreated recurrent PCNSL showed an ORR of $47 \% .^{30}$ Two small retrospective series illustrated activity of a combination therapy of temozolomide and rituximab. ${ }^{32,31}$ The larger series of 15 patients used high-dose rituximab $750 \mathrm{mg} / \mathrm{m}^{2}$ weekly in combination with a week-on/week-off schedule of TMZ $100-200 \mathrm{mg} / \mathrm{m}^{2}$ (rituximab for up to 2 cycles) showed an overall response rate of $53 \% .^{32}$ This prompted an evaluation of this regimen in a prospective trial with planned 40 patients. The study was closed early, after enrollment of 16 patients, due to slow accrual and preliminary analysis suggesting futility. The overall RR was $36 \%{ }^{33}$ Overall, these results show modest activity of TMZ in recurrent PCNSL and this treatment is typically reserved for patients that are not suitable for more aggressive treatment strategies.

\section{Pemetrexed}

Pemetrexed has been an interesting drug to study in recurrent PCNSL as it is an antifolate, similar to MTX. The key difference to MTX is that pemetrexed targets several sites in folate metabolism, involving both purine and pyrimidine metabolism. A prospective trial of pemetrexate monotherapy, at $900 \mathrm{mg} / \mathrm{m}^{2}$ every 3 weeks in 11 patients with relapsed or refractory PCNSL showed an overall response rate of 55\% (OR and PR) and disease control rate, when including $\mathrm{SD}$, of $91 \%{ }^{34}$ A similarly designed prospective trial from China, on 17 patients, using the same regimen, showed a similar ORR of $59 \%$, similar to that reported in the former trial. Survival data and toxicity profile were comparable as well. ${ }^{35}$ A retrospective study of pemetrexed as salvage for not only relapsed PCNSL ( $\mathrm{n}=17$ evaluable PCNSL) but also secondary CNSL (SCNSL; $n=12$ ) illustrated an OR rate of $64.7 \%$ (all CR) and 59.3\% (with 2 $\mathrm{CR}$ ), respectively, further illustrating efficacy of this drug in the setting of relapsed CNS lymphoma. ${ }^{36}$ Treatment of pemetrexed has also been studied in combination with rituximab in a prospective trial with 27 patients, showed an ORR of $62.9 \%$ with $22 \%$ of patients reaching a CR. ${ }^{37}$ Of note, activity of pemetrexed was also confirmed in 12 elderly patients (age $>65$ ) with PCNSL that were felt to be unsuitable for HD-MTX-based induction. ${ }^{38}$ Patient numbers are small, but the findings of an ORR of $83.3 \%$ (4 CR, 6 PR) and mOS of 19.5 months were encouraging. The dose of pemetrexed in this trial was lower, at $600 \mathrm{mg} /$ $\mathrm{m}^{2}$ every 3 weeks. These data suggest that pemetrexed may be a reasonable option for select frail or elderly patients who are not suitable to receive HD-MTX-based therapy for treatment of their PCNSL. ${ }^{38}$

\section{High-Dose Cytarabine Alone or in Combination}

High-dose cytarabine (Ara-C) has been used as single agent and in combination with other agents. ${ }^{39-42}$ In a retrospective analysis on long-term survival of patients with PCNSL, 15 of 31 patients had relapsed after initial CR. Three patients were 


\begin{tabular}{|c|c|c|c|c|c|c|c|c|c|c|c|c|c|c|c|c|}
\hline 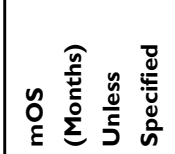 & $\frac{a}{a}$ & $\bar{\gamma}$ & 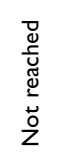 & $\stackrel{\sim}{m}$ & ⿵人丶 & $\infty$ & \pm & 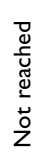 & o. & $\stackrel{\infty}{\wedge}$ & 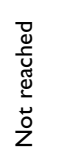 & 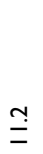 & $\stackrel{n}{\sigma}$ & 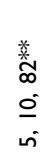 & $\begin{array}{l}" 1 \\
\stackrel{0}{0} \\
\stackrel{E}{\simeq} \stackrel{\circ}{\sigma}\end{array}$ & $\begin{array}{l}\text { "I } \\
\stackrel{O}{E} \\
\stackrel{E}{\simeq} \stackrel{\circ}{\sigma}\end{array}$ \\
\hline
\end{tabular}

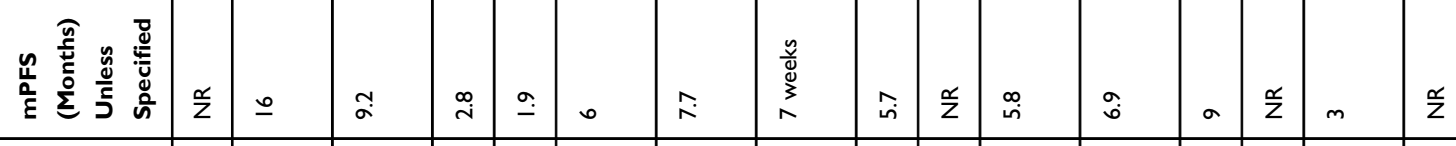

\begin{tabular}{|c|c|c|c|c|c|c|c|c|c|c|c|c|c|c|c|}
\hline $\begin{array}{l}\hat{\varepsilon} \\
\frac{x}{2}\end{array}$ & $\underset{\sim}{\stackrel{\sigma}{\sigma}}$ & $\underset{\sigma}{\stackrel{\varrho}{\sigma}}$ & 4 & $\underset{\sim}{\widehat{o}}$ & $\stackrel{\underline{m}}{=}$ & 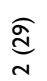 & $\underset{N}{\stackrel{m}{=}}$ & $\underset{\mathrm{m}}{\widehat{\bar{d}}}$ & $\underset{\sim}{\stackrel{00}{N}}$ & 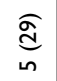 & $\underset{0}{\widehat{o}}$ & $\begin{array}{l}\overline{\bar{y}} \\
=\end{array}$ & 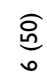 & 誉 & 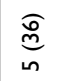 \\
\hline
\end{tabular}

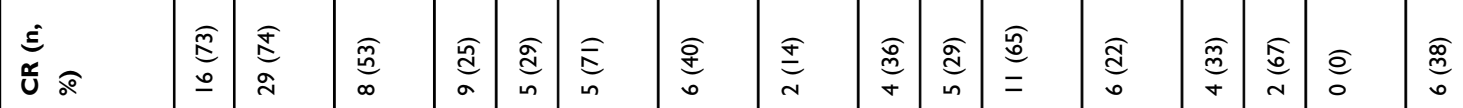

\begin{tabular}{|c|c|c|c|c|c|c|c|c|c|c|c|c|c|c|c|c|}
\hline 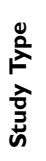 & 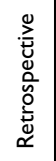 & 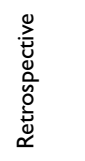 & 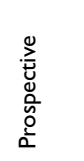 & 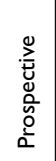 & 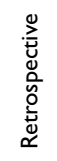 & 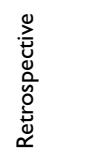 & 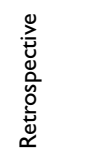 & 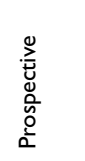 & 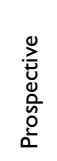 & 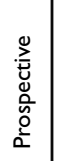 & 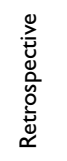 & 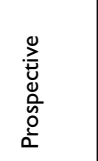 & 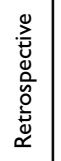 & 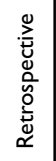 & 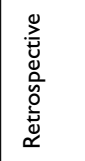 & 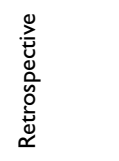 \\
\hline 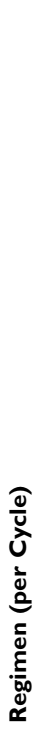 & 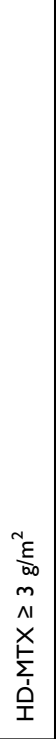 & 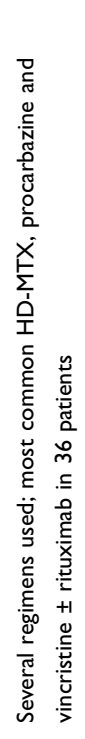 & 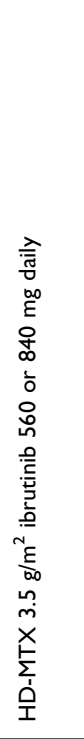 & 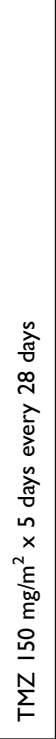 & 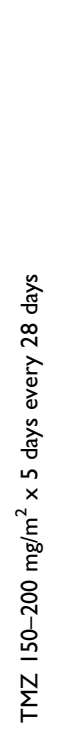 & 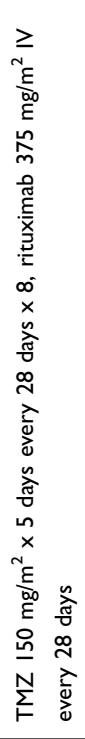 & 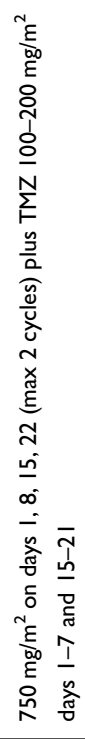 & 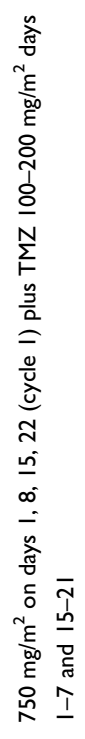 & 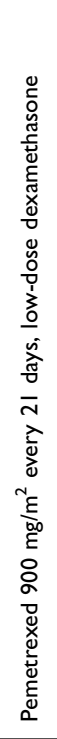 & 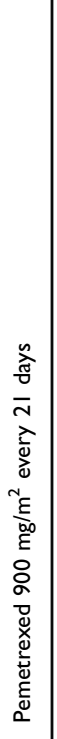 & 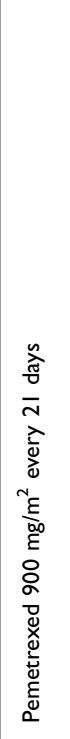 & 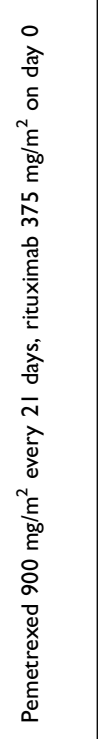 & 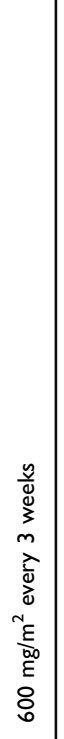 & 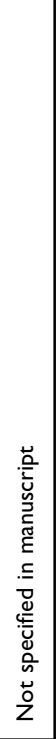 & 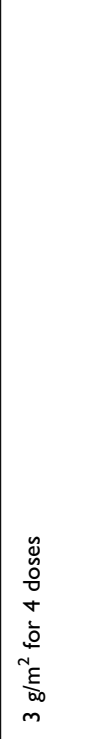 & 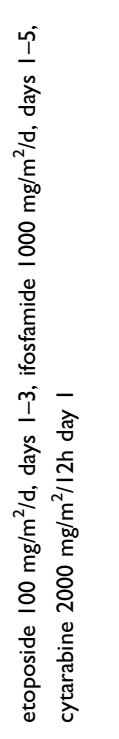 \\
\hline$\stackrel{a}{a}$ & $\begin{array}{l}\stackrel{x}{p} \\
\stackrel{\sum}{\dot{1}} \\
\text { 仓ે }\end{array}$ & 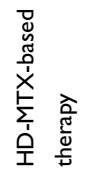 & 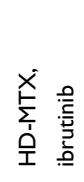 & 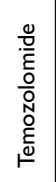 & 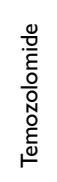 & 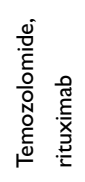 & 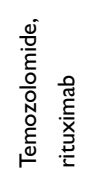 & 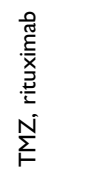 & 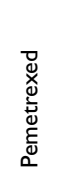 & 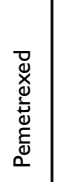 & 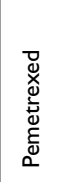 & 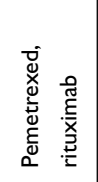 & 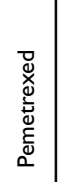 & 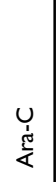 & 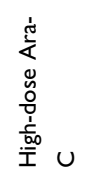 & 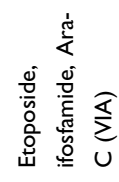 \\
\hline 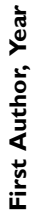 & 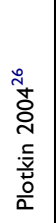 & 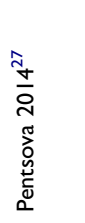 & 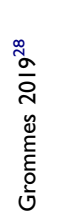 & 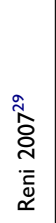 & 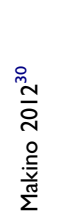 & 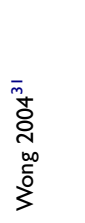 & 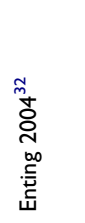 & 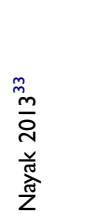 & 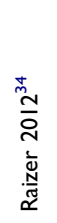 & 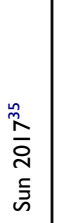 & 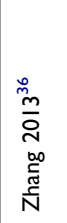 & $\begin{array}{l}\frac{n}{2} \\
\frac{1}{2} \\
\frac{0}{N} \\
\frac{\pi}{N}\end{array}$ & 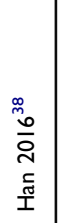 & 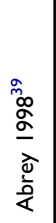 & 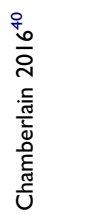 & 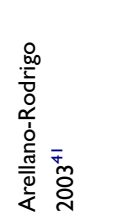 \\
\hline
\end{tabular}




\begin{tabular}{|c|c|c|c|c|c|c|c|c|c|c|c|c|}
\hline 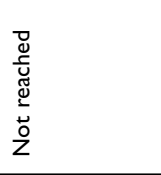 & 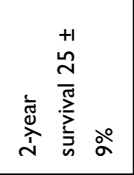 & 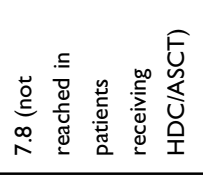 & 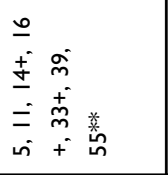 & $\underset{\infty}{+}$ & $\underset{\text { స్ }}{\text { I }}$ & 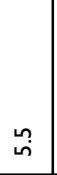 & $\underset{\infty}{\infty}$ & $\hat{m}$ & 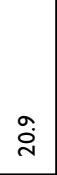 & $\underline{\underline{n}}$ & $\stackrel{\sim}{\underline{\alpha}}$ & $\hat{\Omega}$ \\
\hline$\sigma$ & 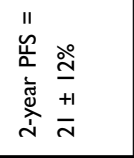 & 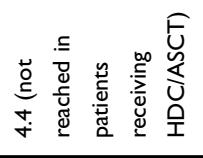 & 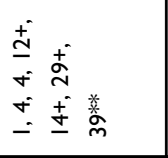 & $\sim$ & $\sim$ & 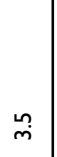 & $\stackrel{\text { m }}{\text { r }}$ & $\bar{i}$ & $\begin{array}{l}\text { 瓷 } \\
\text { in } \\
\text { in }\end{array}$ & $\stackrel{+}{+}$ & $\stackrel{\infty}{+}$ & $\stackrel{\infty}{\sim}$ \\
\hline 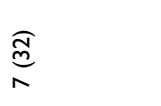 & $\underset{m}{\stackrel{F}{F}}$ & $\begin{array}{l}\mathbb{d} \\
\frac{\tilde{Z}}{z}\end{array}$ & $\underset{\sim}{\stackrel{d}{\mathrm{~d}}}$ & 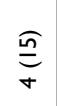 & $\underset{\mathrm{d}}{\widehat{a}}$ & $\underset{\mathrm{m}}{\widehat{d}}$ & $\begin{array}{l}\widehat{\widetilde{d}} \\
\mathrm{~m}\end{array}$ & $\underset{\substack{\hat{e} \\
\underline{v}}}{ }$ & $\stackrel{\widehat{\omega}}{-}$ & $\underset{\substack{a \\
\text { n }}}{n}$ & $\begin{array}{l}\widehat{\tilde{m}} \\
\underline{\underline{n}}\end{array}$ & $\underset{m}{E}$ \\
\hline 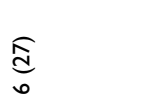 & 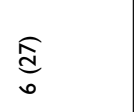 & $\begin{array}{l}\stackrel{\mathscr{Q}}{q} \\
\frac{o}{z}\end{array}$ & $\frac{\sqrt{n}}{\sigma}$ & $\underset{n}{\stackrel{\sigma}{\sigma}}$ & $\underset{m}{\stackrel{d}{d}}$ & 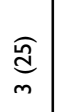 & $\underset{\sim}{\stackrel{n}{2}}$ & 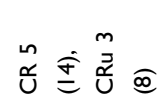 & $\underset{m}{\stackrel{d}{d}}$ & $\underset{\substack{a \\
\text { n }}}{n}$ & $\begin{array}{l}\hat{\sigma} \\
\stackrel{\sigma}{0}\end{array}$ & $\begin{array}{l}\widehat{o} \\
\stackrel{\mathrm{d}}{\mathrm{d}} \\
\underline{m}\end{array}$ \\
\hline in & 8 & t & in & $\bar{n}$ & i̊ & के & $\pi$ & R & 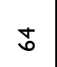 & o & o & $\hat{\sigma}$ \\
\hline$\pi$ & $\pi$ & $\stackrel{\infty}{n}$ & n & $\lambda$ & $\underline{n}$ & $\simeq$ & $\underline{m}$ & $\hat{m}$ & $\simeq$ & $\underline{m}$ & $\approx$ & in \\
\hline 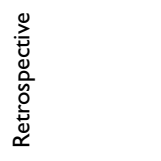 & 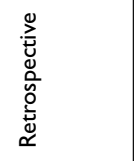 & 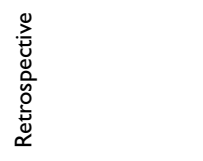 & 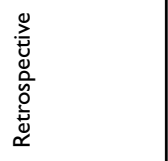 & 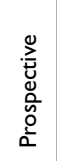 & 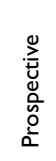 & 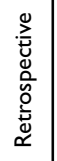 & 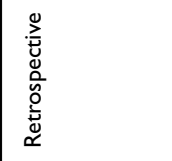 & 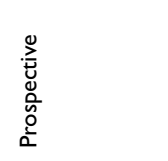 & 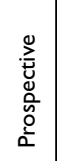 & 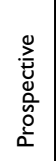 & 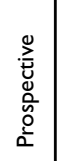 & 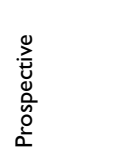 \\
\hline 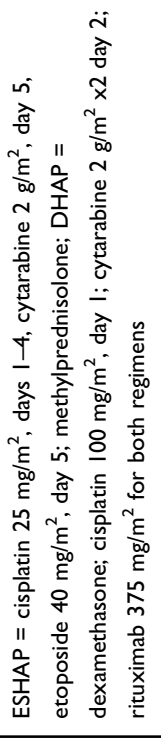 & 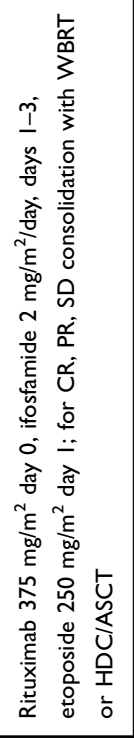 & 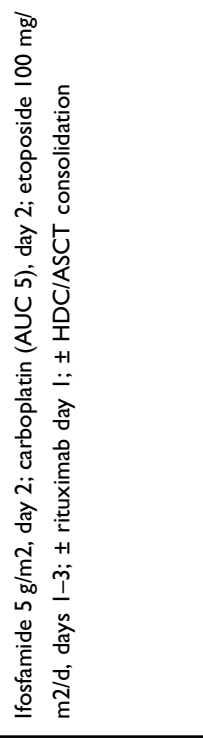 & 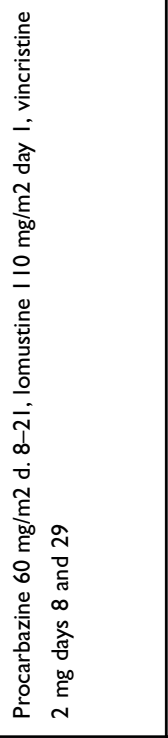 & 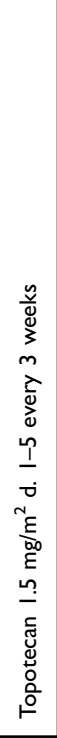 & 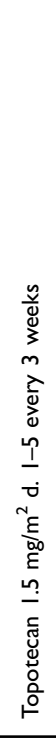 & 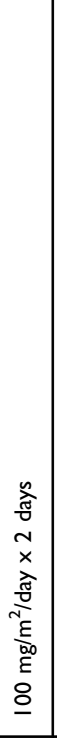 & 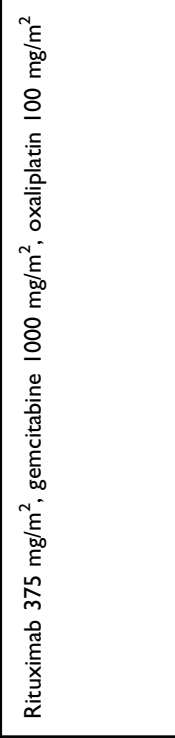 & 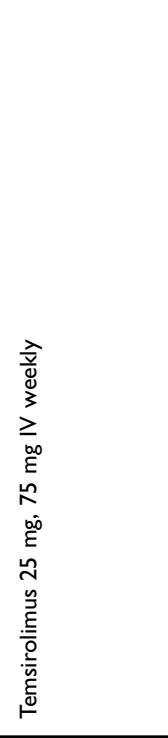 & 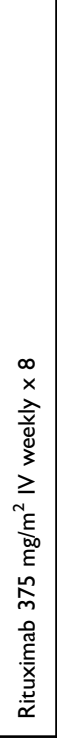 & 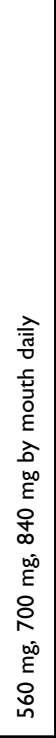 & 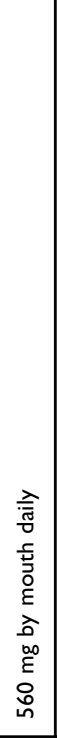 & 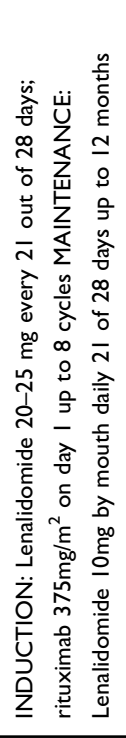 \\
\hline 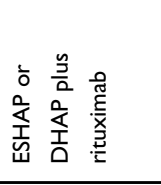 & 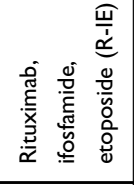 & 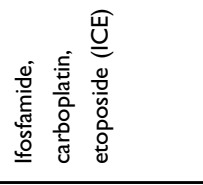 & 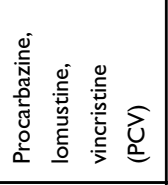 & 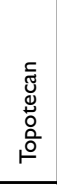 & 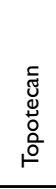 & 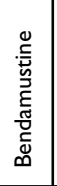 & 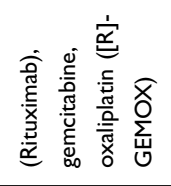 & 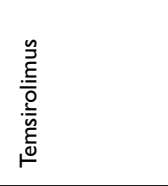 & 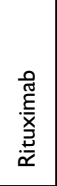 & 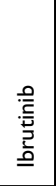 & 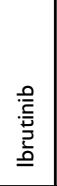 & 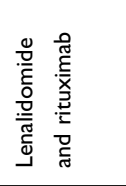 \\
\hline 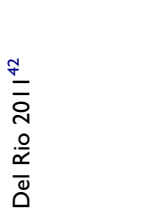 & 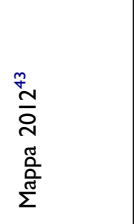 & 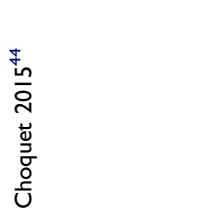 & 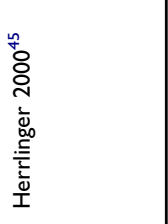 & 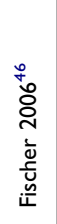 & 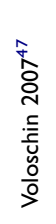 & 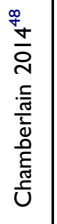 & 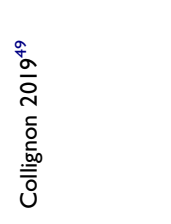 & 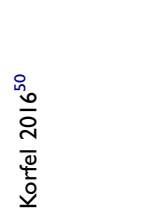 & 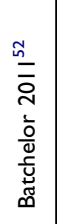 & 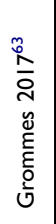 & 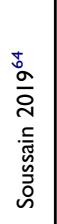 & 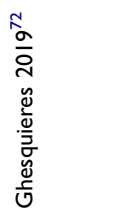 \\
\hline
\end{tabular}




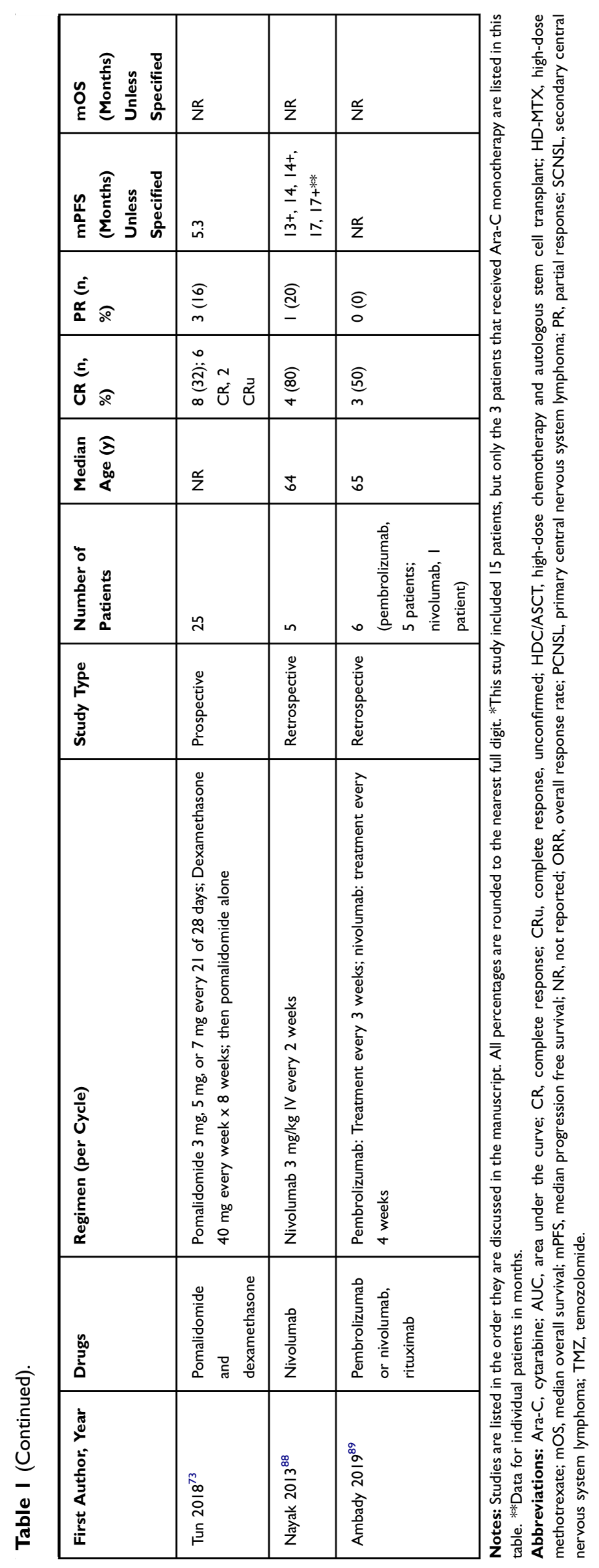


treated with cytarabine alone, yielding a CR in 2 and a PR in 3 patients. Of an additional 4 patients that received cytarabine plus other chemotherapy (the regimens were not further specified in the manuscript), there was 1 patient with a $\mathrm{CR}, 1$ with SD and 2 with PD. ${ }^{39}$ A single agent retrospective study on 14 patients showed a modest response rate of only $35 \%$, all $\mathrm{PR}$, with no $\mathrm{CR}$ reported. ${ }^{40} \mathrm{~A}$ retrospective study on 16 patients with refractory $(n=1)$ or relapsed $(n=15)$ PCNSL that were treated with Etoposide, ifosfamide and cytarabine (VIA) at relapse, showed a CR in $6(37 \%)$ of the patients with durable responses of over 15 months in at least 2 of the patients. ${ }^{41}$ In a French study of 22 patients who had relapsed after initial HD-MTX-based therapy, patients were treated with ESHAP plus rituximab or the DHAP regimen with or without rituximab. In total, 13 patients $(59 \%)$ were chemosensitive to this approach with a CR rate of $27 \%$. As the two latter regimens were combination therapies, it is not possible to assess the impact of cytarabine versus the other regimens studied. $^{42}$

\section{Ifosfamide}

Ifosfamide-based regimens have been studied in $\mathrm{r} / \mathrm{r}$ PCNSL. A patient cohort of 22 consecutive patients with $r / r$ PCNSL were treated with rituximab, ifosfamide and etoposide (R-IE). Prior regimens of 12 of these patients included WBRT and high-dose chemotherapy and autologous transplant. The overall response rate was $41 \%$, with mostly durable responses, however 4 of the responders also underwent consolidation with high-dose chemotherapy and autologous transplant. ${ }^{43}$ A retrospective study of 58 patients with $\mathrm{r} / \mathrm{r}$ PCNSL or vitreoretinal lymphoma, treated at 4 different institutions in France, reported an impressive response rate of $70 \%$ to treatment with ifosfamide, carboplatin and etoposide (ICE). ${ }^{44}$

\section{PCV}

The combination regimen of procarbazine, lomustine and vincristine (PCV) has been studied in a small series of 7 patients with progressive or recurrent PCNSL. ${ }^{45}$ The regimen was of interest as procarbazine and lomustine can cross the $\mathrm{BBB}$ and as this is an established regimen in the treatment of gliomas. The study was published in 2000, prior to the introduction of temozolomide, which then replaced PCV as the main treatment for high-grade astrocytomas. The data show that PCV has evidence of efficacy in recurrent PCNSL. Four patients achieved a CR, 2 a PR and 1 patient progressed. One patient who was only treated with lomustine $110 \mathrm{mg} / \mathrm{m}^{2}$ alone for 9 cycles achieved a PR but lived for 39 months after start of second-line therapy.

\section{Topotecan}

Two prospective studies assessed the efficacy of single-agent topotecan in recurrent PCNSL. ${ }^{46,47}$ The first study reported on 27 patients of which 9 had a response $(\mathrm{ORR}=33 \%){ }^{46}$ A second trial with 15 patients showed similar results, with an ORR of $40 \%{ }^{47}$ Significant myelotoxicity was reported in both regimens, as expected with this drug.

\section{Bendamustine}

Modest single-agent activity was observed in a retrospective study of 12 patients with relapsed PCNSL with half of the patients responding and short mPFS of 3.5 and $\mathrm{mOS}$ of 5.5 months. $^{48}$

\section{Gemcitabine and Oxaliplatin}

A retrospective French study on 13 patients, including older and frailer patients, showed modest activity in $\mathrm{r} / \mathrm{r}$ PCNSL with a combination regimen of rituximab, gemcitabine and oxaliplatin (R-GEMOX). The overall response rate was $38 \%$, with a median PFS of 3.2 months. $^{49}$

\section{Temsirolimus}

A study of 37 patients with refractory or recurrent PCNSL showed activity of single-agent temsirolimus, at $75 \mathrm{mg}$, with an ORR of $54 \%{ }^{50}$ Responses, however, were short lived and the mPFS was only 2.1 months.

\section{Rituximab}

The CD20 targeted monoclonal antibody rituximab has been studied as single agent and in combination in patients with PCNSL. The drug is of interest as most PCNSL are expressing CD20 and as rituximab has led to improved survival in virtually all systemic CD20 positive lymphomas, with overall minimal added toxicity. There has remained controversy regarding the efficacy and the role of rituximab in the treatment of PCNSL. The molecule is too large to cross an intact $\mathrm{BBB}$ and there is concern for lack of its ability to reach tumor cells after closure of the $\mathrm{BBB}$ in patients that are responding to treatment. However, a study of the ${ }^{90}$ Y-labeled anti-CD20 antibody ibritumomab tiuxetan showed intratumoral uptake of the drug in 4 of 6 patients that underwent SPECT imaging, with increasing uptake of up to 5 days after administration. ${ }^{51}$ There were 4 responses reported with this drug, including one durable response of $30+$ months after administration of only one dose of treatment. A small prospective study with single 
agent rituximab $375 \mathrm{mg} / \mathrm{m}^{2}$ in 12 patients with recurrent PCNSL showed an ORR of 36\% (3 CR, 1 PR), illustrating single agent activity of rituximab in this disease. ${ }^{52}$ Several retrospective studies also report improved outcome from the addition of rituximab to the respective standard regimens in newly diagnosed PCNSL, ${ }^{53-57}$ while another study did not identify benefit from the addition of rituximab. ${ }^{58}$ It is of note though that patients in the latter study only received a total of 4 doses of rituximab. The to-date only prospective study randomizing patient to standard therapy with or without rituximab did not find added benefit from rituximab. ${ }^{59}$ This was an intergroup trial of 200 patients with newly diagnosed PCNSL, treated patients with either methotrexate, carmustine, teniposide and prednisone (BMVP) with or without rituximab. However, the number of cycles of rituximab given in this study was limited to 6 , raising the question of whether the amount of rituximab given was sufficient to show a clear benefit between the treatment arms.

\section{Ibrutinib}

Both PCNSL and subsets of DLBCL with a predilection for the CNS are often reliant on chronic active B-cell receptor (BCR) signaling. Ibrutinib is an oral small-molecule that irreversibly inhibits Bruton's tyrosine kinase (BTK) and has selective activity in subsets of DLBCL with a molecular profile resembling PCNSL. ${ }^{60,61}$ Clinical studies in both PCNSL and SCNSL have demonstrated high response rates after ibrutinib monotherapy..$^{28,62,63}$ In a Phase 1 study, ibrutinib monotherapy was given to patients with relapsed or refractory PCNSL and SCNSL. ${ }^{63}$ In this study, $10(77 \%)$ patients with PCNSL responded, including 5 (38\%) who achieved a complete response. The durability of response was only 4.6 months. In SCNSL, 5 (71\%) patients responded including 4 (57\%) complete responses. Overall, the median PFS for SCNSL was 7.4 months, but two patients received ASCT consolidation. A multicenter Phase 2 study of ibrutinib monotherapy in 52 patients with relapsed PCNSL showed that 27 (52\%) patients responded including 10 (19\%) complete responses. However, the median PFS was only 3.3 months (95\% CI, 2-6.4). ${ }^{64}$ Ibrutinib has excellent clinical activity in both PCNSL, and SCNSL, but monotherapy is unlikely to be curative. In another phase 1 study, 18 patients with PCNSL were treated with escalating doses of ibrutinib monotherapy for 14 days as part of a window study design prior to combination of ibrutinib with temozolomide, etoposide, liposomal doxorubicin, dexamethasone, and rituximab (TEDDi-R). ${ }^{62}$ All the chemotherapy agents had demonstrated in vitro synergy with ibrutinib in models of ABC DLBCL. ${ }^{65}$ Of 16 patients evaluable for response, $14(86 \%)$ achieved a complete response, including patients refractory to HD-MTX-based regimens. Notably, the 2-year durable CR rate was $66.7 \%$ (95\% CI: 28.2-87.8). ${ }^{66}$ A number of patients on this study developed serious aspergillosis infections when no antifungal prophylaxis was given, however. Since durable remissions were observed on this study, the regimen is undergoing further study with concomitant antifungal prophylaxis in both PCNSL and SCNSL. In a phase 1B study, ibrutinib has also been added to HD-MTX and rituximab in 15 patients with PCNSL and SCNSL. ${ }^{28}$ In PCNSL, 8 (89\%) patients responded, including $6(67 \%)$ complete responses. In SCNSL, 4 (67\%) patients responded and $2(33 \%)$ achieved a complete response. The durability of response to the regimen is unknown since responding patients were allowed treatment with ASCT consolidation.

\section{Immunomodulatory Agents}

Another class of targeted agents for PCNSL and SCNSL are the oral immunomodulatory agents, lenalidomide and pomalidomide. $^{67}$ These agents use multiple mechanisms, including direct cytotoxic effects and effects on the tumor microenvironment. Lenalidomide binds cereblon and downregulates IRF4 through degradation of Ikaros transcription factors. ${ }^{68}$ IRF4 is a direct target of NF-KB transcription factors induced by B-cell receptor (BCR) signaling and is overexpressed in most cases of PCNSL. ${ }^{69}$ Lenalidomide has single-agent activity in both PCNSL $^{70,71}$ and SCNSL. ${ }^{70}$ In a phase 1 study of lenalidomide in PCNSL and SCNSL, 6 (86\%) patients with PCNSL responded including 1 (14\%) complete response. In SCNSL, 4 (57\%) responses were observed, including 2 (29\%) complete responses. Immunomodulatory agents have also been tested as combination therapy for relapsed or refractory PCNSL. ${ }^{72,73}$ In a multicenter phase 2 study, 50 patients with relapsed or refractory PCNSL received lenalidomide and rituximab followed by lenalidomide monotherapy. ${ }^{72}$ The overall response rate was $32.0 \%$ (95\% CI, 21.9-51.2) including 13 (29\%) complete responses. However, the median PFS was only 7.8 months (95\% CI, 3.9-11.3). In a multicenter phase 1 study, pomalidomide was tested in combination with dexamethasone followed by pomalidomide monotherapy. ${ }^{73}$ In 25 evaluable patients, the overall response rate was $48 \%(95 \% \mathrm{CI}$, 28-69) including $8(32 \%)$ patients who achieved a complete response. The median PFS was 5.3 months (95\% CI, 3.7-16.6). Immunomodulatory agents are 
rational-targeted agents for CNS lymphomas, but will be most effective as combination therapy.

\section{Hematopoietic Stem Cell Transplantation}

High dose chemotherapy (HDC) followed by autologous stem cell transplantation (ASCT) is a standard approach for younger fit patients with relapsed DLBCL that is sensitive to salvage chemotherapy. ASCT is associated with long-term disease control in about half of patients and the treatment-related mortality is low at $1-3 \%{ }^{74,75}$ Durable disease-free control is described with ASCT in patients with SCNSL, though its role in PCNSL is less well-defined. ${ }^{76}$ In one of the earliest retrospective reports describing ASCT in 20 patients with relapsed or refractory PCNSL, the 3-year probability of event-free (EFS) and overall survival (OS) were $53 \%$ and $64 \%$, respectively. ${ }^{77}$ These promising findings led to a multicenter phase II study evaluating HDC with thiotepa, busulfan, and cyclophosphamide (TBC) with ASCT in 27 patients, including 15 who were chemosensitive (12 CR, $3 \mathrm{PR}$ ) to salvage high-dose cytarabine and etoposide and 12 who were chemorefractory. ${ }^{78}$ Post-ASCT, 26 patients were in CR and one had progressive disease. At a median follow-up of 36 months, the median OS was not reached for the chemosensitive patients compared with 18.3 months for the chemorefractory group. A retrospective study from Memorial Sloan Kettering Cancer Center evaluating the same TBC conditioning regimen in 17 patients with $\mathrm{CNS}$ lymphoma (8 PCNSL, 9 SCNSL) had equally remarkable findings with estimated 3-year PFS and OS of 93\%. ${ }^{79}$ Notably all patients achieved CR with salvage therapy; chemorefractory patients were not transplanted. A more recent German Cooperative Group Study studied salvage induction with rituximab, high-dose cytarabine and thiotepa followed by conditioning with rituximab, carmustine, and thiotepa in 39 patients with relapsed PCNSL. ${ }^{80}$ While the results were less encouraging with a 2-year PFS of $46 \%$ at a median follow-up of 45 months, only 4 patients were in CR pre-ASCT. Twenty-two (56\%) achieved CR post-ASCT. These findings underscore the importance of chemosensitive disease. Thiotepa seems to be an essential component of conditioning regimens for PCNSL. Data from a Japanese Transplant Registry revealed that HDC containing thiotepa was a significant factor for PFS on multivariate analysis. ${ }^{81}$ Ongoing shortages of thiotepa may negatively impact outcomes.

HDC with ASCT is a reasonable approach for young, fit patients with chemosensitive disease and may be associated with superior outcomes compared with other available salvage options. Indeed age may be less relevant than fitness, with a recent retrospective study demonstrating similar promising outcomes in elderly patients. ${ }^{82}$ Among 37 patients with a median age of 67 (range 65-77) who underwent ASCT in the second (or greater) line setting, the two year PFS and OS were 54\% and $65.6 \%$, respectively. The treatment-related mortality was low at $3.8 \%$. With several efforts underway to determine if frontline consolidation with ASCT will become standard, the future relevance of ASCT in the relapsed setting remains to be seen. We anxiously await the results of the randomized multicenter trial comparing ASCT to an intensive polychemotherapy approach (CALGB 51,101; NCT01511562). Patients who relapse following upfront ASCT may be candidates for allogeneic transplant. ${ }^{83}$ Small case reports demonstrating successful outcomes with allogeneic transplant suggest a graft versus lymphoma benefit despite the previously assumed immune privileged environment of the CNS. ${ }^{84,85}$

\section{Novel Immunotherapy Approaches}

A gained understanding of CNS immune trafficking ${ }^{86,87}$ has opened avenues for immunotherapy in CNS lymphoma. PCNSL has a unique genetic landscape that is characterized by frequent PD-L1-L2 copy gains along with rare translocations involving the PD-1 ligand loci. ${ }^{16}$ A small series studying PD-1 blockade with nivolumab was encouraging with $100 \%$ overall response rate (3 CR) among 4 patients with relapsed/refractory PCNSL. ${ }^{88}$ A patient series of 6 patients who received PD-1 inhibitor therapy in combination with rituximab showed an ORR of $50 \%$ (3 CR). ${ }^{89}$ Results from multicenter trials with nivolumab and pembrolizumab will be forthcoming (NCT02857426, NCT03255018). Anti-CD19 CAR-T cells are proving effective in SCNSL. ${ }^{90,91}$ Administration of a fourth-generation dual CD19/CD70 CAR-T to a patient with relapsed PCNSL led to disease-free survival which has been maintained with more than 17 months follow-up. ${ }^{92}$ Given that increased neurotoxicity has not been described in this single patient with CNS disease, expansion of CAR-T to patients with PCNSL is likely to be studied further.

\section{Discussion}

There is an increasing number of treatment options for patients with refractory or recurrent PCNSL, many of which did not exist just 20 years ago. However, there is currently no uniform standard of care, and the relatively 
large number of treatment options, which are all based on relatively small trials, makes the choice of treatment challenging. In addition, there are significant differences in institutional preferences.

There are, however, several unifying conclusions that we feel can be derived from the currently available data on systemic treatments in refractory or recurrent PCNSL:

- Patients should be considered for enrollment in clinical trials as there is a need for better treatment options for patients with recurrent PCNSL and as there is no clear standard of care.

- If no clinical trial is available, then patients who had a durable remission after initial induction with HDMTX-based therapy, should be considered for a rechallenge with HD-MTX-based therapy. For patients suitable for consolidation with autologous stem cell transplant, this option should be considered based on high rates of durable responses that have been reported. Non-MTX-based combination regimens, including TEDDi-R, are also being studied in this patient population, but should not be given off trial until further safety data are available.

- In patients not suitable for HD-MTX-based therapy, other single agent or combination regimens with reasonable response rates (Table 1) should be considered. These include patients with severely reduced renal function and patients that have progressed on or shortly after HD-MTX-based treatment. Favorable response rates have been reported with ibrutinib, lenalidomide, pomalidomide, pemetrexed and TMZ. The duration of remissions to these agents, however, is often short. The role of rituximab has not yet been conclusively answered in this setting.

Clinical research in PCNSL is challenging. While response rates and survival in this disease compare favorably to other high-grade malignancies of the CNS, they compare unfavorably to outcomes in patients with many systemic high-grade B-cell lymphomas. PCNSL is a rare disease. Enrollment in prospective clinical trials is therefore strongly encouraged. However, many patients, especially the growing number of older and frail patients with PCNSL, may not be candidates for aggressive treatment regimens or autologous transplant. This also includes patients unsuitable for treatment with HDMTX. There may be a window of opportunity to study nonMTX-based regimens and more indolent treatments in this patient group. As PCNSL is rare, there should also be an emphasis on the collection of real-life data through institutional and collaborative databases to capture important outcome data related to the different treatment approaches for this disease. Only few centers have large enough PCNSL clinical volumes to conclusively evaluate efficacy of certain treatments as a single institution and collaboration between institutions should be encouraged.

\section{Funding}

This study was supported by the Sidney Kimmel Comprehensive Cancer Center core grant, P30CA006973.

\section{Disclosure}

Dr Matthias Holdhoff reports grant support from the National Cancer Institute (NCI), during the conduct of the study; advisory board or consultative work for Celgene, AbbVie, Inc., NewLink Genetics, BTG International Ltd., and DP Clinical, Inc., outside the submitted work. Dr Nina Wagner-Johnston is on the advisory board for ADC Therapeutics, CALIB-R, Verastem, Bayer, Gilead, and JUNO, outside the submitted work. The authors report no other conflicts of interest in this work.

\section{References}

1. Ostrom QT, Gittleman H, Truitt G, Boscia A, Kruchko C, BarnholtzSloan JS. CBTRUS statistical report: primary brain and other central nervous system tumors diagnosed in the united states in 2011-2015. Neuro Oncol. 2018;20(suppl_4):ivliv86. doi:10.1093/neuonc/noy131

2. Grommes C, DeAngelis LM. Primary CNS lymphoma. J Clin Oncol. 2017;35(21):2410-2418. doi:10.1200/JCO.2017.72.7602

3. Hoffman S, Propp JM, McCarthy BJ. Temporal trends in incidence of primary brain tumors in the united states, 19851999. Neuro Oncol. 2006;8(1):27-37. doi:10.1215/S1522851705000323

4. Villano JL, Koshy M, Shaikh H, Dolecek TA, McCarthy BJ. Age, gender, and racial differences in incidence and survival in primary CNS lymphoma. Br J Cancer. 2011;105(9):1414-1418. doi:10.1038/bjc.2011.357

5. Shiels MS, Pfeiffer RM, Besson C, et al. Trends in primary central nervous system lymphoma incidence and survival in the U. $S \mathrm{Br}$ J Haematol. 2016;174(3):417-424. doi:10.1111/bjh.14073

6. Calimeri T, Lopedote P, Ferreri AJM. Risk stratification and management algorithms for patients with diffuse large B-cell lymphoma and CNS involvement. Annals of Lymphoma. 2019;3:7. doi:10.21037/aol.2019.06.01

7. Bernstein SH, Unger JM, Leblanc M, Friedberg J, Miller TP, Fisher RI. Natural history of CNS relapse in patients with aggressive non-hodgkin's lymphoma: a 20-year follow-up analysis of SWOG 8516 - the southwest oncology group. J Clin Oncol. 2009;27 (1):114-119. doi:10.1200/JCO.2008.16.8021

8. Wight JC, Yue M, Keane C, et al. Outcomes of synchronous systemic and central nervous system (CNS) involvement of diffuse large B-cell lymphoma are dictated by the CNS disease: a collaborative study of the australasian lymphoma alliance. Br J Haematol. 2019;187 (2):174-184. doi:10.1111/bjh.16064

9. Alizadeh AA, Eisen MB, Davis RE, et al. Distinct types of diffuse large B-cell lymphoma identified by gene expression profiling. Nature. 2000;403(6769):503-511. doi:10.1038/35000501 
10. Rosenwald A, Wright G, Chan WC, et al. The use of molecular profiling to predict survival after chemotherapy for diffuse large-B-cell lymphoma. $N$ Engl J Med. 2002;346(25):1937-1947. doi:10.1056/NEJMoa012914

11. Lenz G, Wright GW, Emre NC, et al. Molecular subtypes of diffuse large B-cell lymphoma arise by distinct genetic pathways. Proc Natl Acad Sci U S A. 2008;105(36):13520-13525. doi:10.1073/ pnas.0804295105

12. Villa D, Tan KL, Steidl C, et al. Molecular features of a large cohort of primary central nervous system lymphoma using tissue microarray. Blood $A d v$. 2019;3(23):3953-3961. doi:10.1182/bloodadvances.2019000989

13. Fukumura K, Kawazu M, Kojima S, et al. Genomic characterization of primary central nervous system lymphoma. Acta Neuropathol. 2016;131(6):865-875. doi:10.1007/s00401-016-1536-2

14. Braggio E, Van Wier S, Ojha J, et al. Genome-wide analysis uncovers novel recurrent alterations in primary central nervous system lymphomas. Clin Cancer Res. 2015;21(17):3986-3994. doi:10.1158/ 1078-0432.CCR-14-2116

15. Bruno A, Boisselier B, Labreche K, et al. Mutational analysis of primary central nervous system lymphoma. Oncotarget. 2014;5 (13):5065-5075. doi:10.18632/oncotarget.2080

16. Chapuy B, Roemer MG, Stewart C, et al. Targetable genetic features of primary testicular and primary central nervous system lymphomas. Blood. 2016;127(7):869-881. doi:10.1182/blood-2015-10-673236

17. Schmitz R, Wright GW, Huang DW, et al. Genetics and pathogenesis of diffuse large B-cell lymphoma. $N$ Engl $J$ Med. 2018;378 (15):1396-1407. doi:10.1056/NEJMoa1801445

18. Cai Q, Fang Y, Young KH. Primary central nervous system lymphoma: molecular pathogenesis and advances in treatment. Transl Oncol. 2019;12(3):523-538. doi:10.1016/j.tranon.2018.11.011

19. Illerhaus G, Schorb E, Kasenda B. Novel agents for primary central nervous system lymphoma: evidence and perspectives. Blood. 2018;132(7):681-688. doi:10.1182/blood-2018-01-791558

20. Grommes C, Nayak L, Tun HW, Batchelor TT. Introduction of novel agents in the treatment of primary CNS lymphoma. Neuro Oncol. 2019;21(3):306-313. doi:10.1093/neuonc/noy193

21. Ambady P, Holdhoff M, Bonekamp D, Wong F, Grossman SA. Late relapses in primary CNS lymphoma after complete remissions with high-dose methotrexate monotherapy. CNS Oncol. 2015;4 (6):393-398. doi:10.2217/cns.15.34

22. Nayak L, Hedvat C, Rosenblum MK, Abrey LE, DeAngelis LM. Late relapse in primary central nervous system lymphoma: clonal persistence. Neuro Oncol. 2011;13(5):525-529. doi:10.1093/neuonc/ nor014

23. Langner-Lemercier S, Houillier C, Soussain C, et al. Primary CNS lymphoma at first relapse/progression: characteristics, management, and outcome of 256 patients from the French LOC network. Neuro Oncol. 2016;18(9):1297-1303. doi:10.1093/neuonc/now033

24. Ferreri AJM, Holdhoff M, Nayak L, Rubenstein JL. Evolving treatments for primary central nervous system lymphoma. Am Soc Clin Oncol Educ Book. 2019;39:454-466. doi:10.1200/EDBK_242547

25. Yahalom J, Illidge $\mathrm{T}$, Specht L, et al., International Lymphoma Radiation Oncology Group. Modern radiation therapy for extranodal lymphomas: field and dose guidelines from the International Lymphoma radiation oncology group. Int J Radiat Oncol Biol Phys. 2015;92(1):11-31. doi:10.1016/j.ijrobp.2015.01.009

26. Plotkin SR, Betensky RA, Hochberg FH, et al. Treatment of relapsed central nervous system lymphoma with high-dose methotrexate. Clin Cancer Res. 2004;10(17):5643-5646. doi:10.1158/1078-0432.CCR-04-0159

27. Pentsova E, Deangelis LM, Omuro A. Methotrexate re-challenge for recurrent primary central nervous system lymphoma. J Neurooncol. 2014;117(1):161-165. doi:10.1007/s11060-014-1370-0

28. Grommes C, Tang SS, Wolfe J, et al. Phase $1 \mathrm{~b}$ trial of an ibrutinib-based combination therapy in recurrent/refractory CNS lymphoma. Blood. 2019;133(5):436-445. doi:10.1182/blood-201809-875732
29. Reni M, Zaja F, Mason W, et al. Temozolomide as salvage treatment in primary brain lymphomas. Br J Cancer. 2007;96(6):864-867. doi:10.1038/sj.bjc. 6603660

30. Makino K, Nakamura H, Hide T, Kuratsu J. Salvage treatment with temozolomide in refractory or relapsed primary central nervous system lymphoma and assessment of the MGMT status. $J$ Neurooncol. 2012;106(1):155-160. doi:10.1007/s11060-011-0652-z

31. Wong ET, Tishler R, Barron L, Wu JK. Immunochemotherapy with rituximab and temozolomide for central nervous system lymphomas. Cancer. 2004;101(1):139-145. doi:10.1002/cncr.20339

32. Enting RH, Demopoulos A, DeAngelis LM, Abrey LE. Salvage therapy for primary CNS lymphoma with a combination of rituximab and temozolomide. Neurology. 2004;63(5):901-903. doi:10.1212/01. WNL.0000137050.43114.42

33. Nayak L, Abrey LE, Drappatz J, et al. Multicenter phase II study of rituximab and temozolomide in recurrent primary central nervous system lymphoma. Leuk Lymphoma. 2013;54(1):58-61. doi:10.31 09/10428194.2012.698736

34. Raizer JJ, Rademaker A, Evens AM, et al. Pemetrexed in the treatment of relapsed/refractory primary central nervous system lymphoma. Cancer. 2012;118(15):3743-3748. doi:10.1002/cncr. 26709

35. Sun Y, Wang Y, Han S, et al. Efficacy and safety of pemetrexed on recurrent primary central nervous system lymphomas in china: A prospective study. Onco Targets Ther. 2017;10:2595-2600. doi:10.2147/OTT.S134684

36. Zhang JP, Lee EQ, Nayak L, et al. Retrospective study of pemetrexed as salvage therapy for central nervous system lymphoma. J Neurooncol. 2013;115(1):71-77. doi:10.1007/s11060-013-1196-1

37. Zhao HT, Chen J, Shi SB, Tian J, Tao RJ. Pemetrexed plus rituximab as second-line treatment for primary central nervous system lymphoma. Med Oncol. 2015;32(1):351-014-0351-7. doi:10.1007/ s12032-014-0351-7

38. Han S, Wang M, Liu B, Yu J. Pemetrexed for primary central nervous system lymphoma in the elderly. Clin Transl Oncol. 2016;18(2):138143. doi:10.1007/s12094-015-1345-4

39. Abrey LE, DeAngelis LM, Yahalom J. Long-term survival in primary CNS lymphoma. J Clin Oncol. 1998;16(3):859-863. doi:10.1200/ JCO.1998.16.3.859

40. Chamberlain MC. High-dose cytarabine salvage therapy for recurrent primary CNS lymphoma. J Neurooncol. 2016;126(3):545-550. doi:10.1007/s11060-015-1994-8

41. Arellano-Rodrigo E, Lopez-Guillermo A, Bessell EM, Nomdedeu B, Montserrat E, Graus F. Salvage treatment with etoposide (VP-16), ifosfamide and cytarabine (ara-C) for patients with recurrent primary central nervous system lymphoma. Eur J Haematol. 2003;70 (4):219-224. doi:10.1034/j.1600-0609.2003.00045.x

42. Del Rio MS, Choquet S, Hoang-Xuan K, et al. Platine and cytarabine-based salvage treatment for primary central nervous system lymphoma. J Neurooncol. 2011;105(2):409-414. doi:10.1007/ s11060-011-0608-3

43. Mappa S, Marturano E, Licata G, et al. Salvage chemoimmunotherapy with rituximab, ifosfamide and etoposide (R-IE regimen) in patients with primary CNS lymphoma relapsed or refractory to high-dose methotrexate-based chemotherapy. Hematol Oncol. 2013;31(3):143-150. doi:10.1002/hon.2037

44. Choquet S, Grenier A, Houillier C, et al. Very high efficiency of ICE (Ifosfamide-Carboplatin-Etoposide) in Relapse/Refractory (R/R) Primary Central Nervous System (PCNSL) and Vitreo-Retinal (VRL) Non Hodgkin Lymphoma. A LOC network multicenter retrospective study on 58 cases. Blood. 2015;126(23):1524.

45. Herrlinger U, Brugger W, Bamberg M, Kuker W, Dichgans J, Weller M. PCV salvage chemotherapy for recurrent primary CNS lymphoma. Neurology. 2000;54(8):1707-1708. doi:10.1212/ WNL.54.8.1707 
46. Fischer L, Thiel E, Klasen HA, et al. Prospective trial on topotecan salvage therapy in primary CNS lymphoma. Ann Oncol. 2006;17 (7):1141-1145. doi:10.1093/annonc/mdl070

47. Voloschin AD, Betensky R, Wen PY, Hochberg F, Batchelor T. Topotecan as salvage therapy for relapsed or refractory primary central nervous system lymphoma. $J$ Neurooncol. 2008;86 (2):211-215. doi:10.1007/s11060-007-9464-6

48. Chamberlain MC. Salvage therapy with bendamustine for methotrexate refractory recurrent primary CNS lymphoma: A retrospective case series. J Neurooncol. 2014;118(1):155-162. doi:10.1007/s11060-014$1411-8$

49. Collignon A, Houillier C, Ahle G, et al. R)-GEMOX chemotherapy for unfit patients with refractory or recurrent primary central nervous system lymphoma: a LOC study. Ann Hematol. 2019;98(4):915-922. doi:10.1007/s00277-018-3564-6

50. Korfel A, Schlegel U, Herrlinger U, et al. Phase II trial of temsirolimus for Relapsed/Refractory primary CNS lymphoma. J Clin Oncol. 2016;34(15):1757-1763. doi:10.1200/JCO.2015.64.9897

51. Maza S, Kiewe P, Munz DL, et al. First report on a prospective trial with yttrium-90-labeled ibritumomab tiuxetan (zevalin) in primary CNS lymphoma. Neuro Oncol. 2009;11(4):423-429. doi:10.1215/ 15228517-2008-108

52. Batchelor TT, Grossman SA, Mikkelsen T, Ye X, Desideri S, Lesser GJ. Rituximab monotherapy for patients with recurrent primary CNS lymphoma. Neurology. 2011;76(10):929-930. doi:10.1212/WNL.0b013e31820f2d94

53. Gregory G, Arumugaswamy A, Leung T, et al. Rituximab is associated with improved survival for aggressive B cell CNS lymphoma. Neuro Oncol. 2013;15(8):1068-1073. doi:10.1093/neuonc/not032

54. Holdhoff M, Ambady P, Abdelaziz A, et al. High-dose methotrexate with or without rituximab in newly diagnosed primary CNS lymphoma. Neurology. 2014;83(3):235-239. doi:10.1212/WNL.00 00000000000593

55. Birnbaum T, Stadler EA, von Baumgarten L, Straube A. Rituximab significantly improves complete response rate in patients with primary CNS lymphoma. J Neurooncol. 2012;109(2):285-291. doi:10.1007/s11060-012-0891-7

56. Mocikova H, Pytlik R, Sykorova A, et al. Role of rituximab in treatment of patients with primary central nervous system lymphoma: a retrospective analysis of the czech lymphoma study group registry. Leuk Lymphoma. 2016;57(12):2777-2783. doi:10.3109/10428194. 2016.1167203

57. Houillier $\mathrm{C}$, Ghesquières $\mathrm{H}$, Chabrot $\mathrm{C}$, et al. Rituximab, methotrexate, procarbazine, vincristine and intensified cytarabine consolidation for primary central nervous system lymphoma (PCNSL) in the elderly: a LOC network study. J Neurooncol. 2017;133(2):315-320. doi:10.1007/s11060-017-2435-7

58. Kansara R, Shenkier TN, Connors JM, et al. Rituximab with high-dose methotrexate in primary central nervous system lymphoma. Am J Hematol. 2015;90(12):1149-1154. doi:10.1002/ ajh. 24204

59. Bromberg JEC, Issa $\mathrm{S}$, Bakunina $\mathrm{K}$, et al. Rituximab in patients with primary CNS lymphoma (HOVON 105/ALLG NHL 24): A randomised, open-label, Phase 3 intergroup study. Lancet Oncol. 2019;20(2):216-228. doi:10.1016/S1470-2045(18)30747-2

60. Wilson WH, Young RM, Schmitz R, et al. Targeting B cell receptor signaling with ibrutinib in diffuse large B cell lymphoma. Nat Med. 2015;21(8):922-926. doi:10.1038/nm.3884

61. Winer ES, Ingham RR, Castillo JJ. PCI-32765: A novel Bruton's tyrosine kinase inhibitor for the treatment of lymphoid malignancies. Expert Opin Investig Drugs. 2012;21(3):355-361. doi:10.1517/ 13543784.2012.656199

62. Lionakis MS, Dunleavy K, Roschewski M, et al. Inhibition of B cell receptor signaling by ibrutinib in primary CNS lymphoma. Cancer Cell. 2017;31(6):833-843.e5. doi:10.1016/j.ccell.2017.04.012
63. Grommes C, Pastore A, Palaskas N, et al. Ibrutinib unmasks critical role of Bruton tyrosine kinase in primary CNS lymphoma. Cancer Discov. 2017;7(9):1018-1029. doi:10.1158/2159-8290.CD-17-0613

64. Soussain $\mathrm{C}$, Choquet $\mathrm{S}$, Blonski $\mathrm{M}$, et al. Ibrutinib monotherapy for relapse or refractory primary CNS lymphoma and primary vitreoretinal lymphoma: final analysis of the phase II 'proof-of-concept' iLOC study by the lymphoma study association (LYSA) and the french oculo-cerebral lymphoma (LOC) network. Eur $J$ Cancer. 2019;117:121-130. doi:10.1016/j.ejca.2019.05.024

65. Mathews Griner LA, Guha R, Shinn P, et al. High-throughput combinatorial screening identifies drugs that cooperate with ibrutinib to kill activated B-cell-like diffuse large B-cell lymphoma cells. Proc Natl Acad Sci U S A. 2014;111(6):2349-2354. doi:10.1073/pnas.1311846111

66. Roschewski M, Lionakis MS, Melani C, et al. Dose-adjusted teddi- $\mathrm{R}$ induces durable compete remissions in relapsed and refractory primary CNS lymphoma. Blood. 2018;132:4195. doi:10.1182/ blood-2018-99-112184

67. Tageja N. Lenalidomide - current understanding of mechanistic properties. Anticancer Agents Med Chem. 2011;11(3):315-326. doi:10.2174/187152011795347487

68. Zhang LH, Kosek J, Wang M, Heise C, Schafer PH, Chopra R. Lenalidomide efficacy in activated B-cell-like subtype diffuse large B-cell lymphoma is dependent upon IRF4 and cereblon expression. Br J Haematol. 2013;160(4):487-502. doi:10.1111/bjh. 12172

69. Camilleri-Broet S, Criniere E, Broet P, et al. A uniform activated B-cell-like immunophenotype might explain the poor prognosis of primary central nervous system lymphomas: analysis of 83 cases. Blood. 2006;107(1):190-196. doi:10.1182/blood-2005-03-1024

70. Rubenstein JL, Geng H, Fraser EJ, et al. Phase 1 investigation of lenalidomide/rituximab plus outcomes of lenalidomide maintenance in relapsed CNS lymphoma. Blood Adv. 2018;2(13):1595-1607. doi:10.1182/bloodadvances.2017014845

71. Houillier C, Choquet S, Touitou V, et al. Lenalidomide monotherapy as salvage treatment for recurrent primary CNS lymphoma. Neurology. 2015;84(3):325-326. doi:10.1212/WNL.0000000000001158

72. Ghesquieres $\mathrm{H}$, Chevrier M, Laadhari $\mathrm{M}$, et al. Lenalidomide in combination with intravenous rituximab (REVRI) in relapsed/refractory primary CNS lymphoma or primary intraocular lymphoma: A multicenter prospective 'proof of concept' phase II study of the french oculo-cerebral lymphoma (LOC) network and the lymphoma study association (LYSA) dagger. Ann Oncol. 2019;30(4):621-628. doi:10.1093/annonc/mdz032

73. Tun HW, Johnston PB, DeAngelis LM, et al. Phase 1 study of pomalidomide and dexamethasone for relapsed/refractory primary CNS or vitreoretinal lymphoma. Blood. 2018;132(21):2240-2248. doi:10.1182/blood-2018-02-835496

74. Philip T, Guglielmi C, Hagenbeek A, et al. Autologous bone marrow transplantation as compared with salvage chemotherapy in relapses of chemotherapy-sensitive non-Hodgkin's lymphoma. $N$ Engl $J$ Med. 1995;333:1540-1545. doi:10.1056/NEJM199512073332305

75. Gisselbrecht C, Glass B, Mounier N, et al. Salvage regimens with autologous transplantation for relapsed large B-cell lymphoma in the rituximab era. J Clin Oncol. 2010;28:4184 4190. doi:10.1200/JCO.2010.28.1618

76. van Besien K, Przepiorka D, Mehra R, et al. Impact of preexisting CNS involvement on the outcome of bone marrow transplantation in adult hematologic malignancies. J Clin Oncol. 1996;14:3036-3042. doi:10.1200/JCO.1996.14.11.3036

77. Soussain C, Suzan F, Hoang-Xuan K, et al. Results of intensive chemotherapy followed by hematopoietic stem-cell rescue in 22 patients with refractory or recurrent primary CNS lymphoma or intraocular lymphoma. J Clin Oncol. 2001;19(3):742-749. doi:10.1200/JCO.2001.19.3.742

78. Soussain C, Hoang-Xuan K, Taillandier L, et al. Intensive chemotherapy followed by hematopoietic stem-cell rescue for refractory and recurrent primary CNS and intraocular lymphoma: societe francaise de greffe de moelle osseuse-therapie cellulaire. J Clin Oncol. 2008;26 (15):2512-2518. doi:10.1200/JCO.2007.13.5533 
79. Welch MR, Sauter CS, Matasar MJ, et al. Autologous stem cell transplant in recurrent or refractory primary or secondary central nervous system lymphoma using thiotepa, busulfan and cyclophosphamide. Leuk Lymphoma. 2015;56(2):361-367. doi:10.3109/10428194.2014.916800

80. Kasenda B, Ihorst G, Schroers R, et al. High-dose chemotherapy with autologous haematopoietic stem cell support for relapsed or refractory primary CNS lymphoma: a prospective multicentre trial by the German Cooperative PCNSL study group. Leukemia. 2017;31:2623-2629. doi:10.1038/leu.2017.170

81. Kondo E, Ikeda T, Izutsu K, et al. High-dose chemotherapy with autologous stem cell transplantation in primary central nervous system lymphoma: data from the japan society for hematopoietic cell transplantation registry. Biol Blood Marrow Transplant. 2019;25 (5):899-905. doi:10.1016/j.bbmt.2019.01.020

82. Schorb E, Fox CP, Fritsch K, et al. High-dose thiotepa-based chemotherapy with autologous stem cell support in elderly patients with primary central nervous system lymphoma: a European retrospective study. Bone Marrow Transplant. 2017;52:1113-1119. doi:10.1038/bmt.2017.23

83. Mika T, Ladigan S, Baraniskin A, et al. Allogeneic hematopoietic stem cell transplantation for primary central nervous system lymphoma. Haematologica. 2020;105:e160-e163. doi:10.3324/haematol.2019.227199

84. Varadi G, Or R, Kapelushnik J, et al. Graft-versus-lymphoma effect after allogeneic peripheral blood stem cell transplantation for primary central nervous system lymphoma. Leuk Lymphoma. 1999;34 (12):185-190. doi:10.3109/10428199909083396

85. Atilla E, Sahin U, Atilla PA, et al. Allogeneic stem cell transplantation for relapsed primary central nervous system lymphoma: is it feasible? Hematol Oncol Stem Cell Ther. 2019;12(4):220-225. doi:10.1016/j.hemonc.2018.02.001
86. Carson MJ, Doose JM, Melchior B, Schmid CD, Ploix CC. CNS immune privilege: hiding in plain sight. Immunol Rev. 2006;213:48-65. doi:10.1111/j.1600-065X.2006.00441.X

87. Wilson EH, Weninger W, Hunter CA. Trafficking of immune cells in the central nervous system. J Clin Invest. 2010;120(5):1368-1379. doi:10.1172/JCI41911

88. Nayak L, Iwamoto FM, LaCasce A, et al. PD-1 blockade with nivolumab in relapsed/refractory primary central nervous system and testicular lymphoma. Blood. 2017;129(23):3071-3073. doi:10. 1182/blood-2017-01-764209

89. Ambady P, Szidonya L, Firkins J, et al. Combination immunotherapy as a non-chemotherapy alternative for refractory or recurrent CNS lymphoma. Leuk Lymphoma. 2019;60(2):515-518. doi:10.1080/ 10428194.2018.1480771

90. Abramson JS, McGree B, Noyes S, et al. Anti-CD19 CAR T cells in CNS diffuse large-B-cell lymphoma. $N$ Engl J Med. 2017;377 (8):783-784. doi:10.1056/NEJMc1704610

91. Frigault MJ, Dietrich J, Martinez-Lage M, et al. Tisagenlecleucel CAR T-cell therapy in secondary CNS lymphoma. Blood. 2019;134 (11):860-866. doi:10.1182/blood.2019001694

92. Tu S, Zhou X, Guo Z, et al. CD19 and CD70 dual-target chimeric antigen receptor T-cell therapy for the treatment of relapsed and refractory primary central nervous system diffuse large B-cell lymphoma. Front Oncol. 2019;9:1350. doi:10.3389/fonc.2019.01350
OncoTargets and Therapy

\section{Publish your work in this journal}

OncoTargets and Therapy is an international, peer-reviewed, open access journal focusing on the pathological basis of all cancers, potential targets for therapy and treatment protocols employed to improve the management of cancer patients. The journal also focuses on the impact of management programs and new therapeutic

\section{Dovepress}

agents and protocols on patient perspectives such as quality of life, adherence and satisfaction. The manuscript management system is completely online and includes a very quick and fair peer-review system, which is all easy to use. Visit http://www.dovepress.com/ testimonials.php to read real quotes from published authors. 